\title{
Synthesis, Antimicrobial, and Anti-HIV1 Activity of Quinazoline-4(3H)-one Derivatives
}

\author{
K. Vijayakumar, ${ }^{1}$ A. Jafar Ahamed, ${ }^{2}$ and G. Thiruneelakandan ${ }^{3}$ \\ ${ }^{1}$ Department of Chemistry, Sri Angalamman College of Engneering \& Technology, Tiruchirappalli, Tamil Nadu 620 020, India \\ ${ }^{2} P G$ \& Research Department of Chemistry, Jamal Mohamed College, Tiruchirappalli, Tamil Nadu 620 020, India \\ ${ }^{3}$ Department of Microbiology, Srimad Andavan Arts and Science College, Tiruchirappalli, Tamil Nadu 620 005, India
}

Correspondence should be addressed to K. Vijayakumar; knvijaykumar72@gmail.com

Received 8 April 2013; Revised 12 July 2013; Accepted 18 July 2013

Academic Editor: Rassoul Dinarvand

Copyright $\odot 2013$ K. Vijayakumar et al. This is an open access article distributed under the Creative Commons Attribution License, which permits unrestricted use, distribution, and reproduction in any medium, provided the original work is properly cited.

\begin{abstract}
The present investigation aims to synthesize 11 compounds of quinazoline-1 derivatives and to test their antimicrobial and anti-HIV1 activities. A quick-witted method was developed for the synthesis of novel substituted quinazolinone derivatives by summarizing diverse diamines with benzoxazine reactions, and it demonstrated the benefits of typical reactions, handy operation, and outstanding product yields. These compounds were confirmed by elemental analysis, I R, ${ }^{1} \mathrm{H}$ NMR, ${ }^{13} \mathrm{C}$ NMR, and mass spectra. Then antimicrobial and anti-HIV1 activities of the compounds were tested in-vitro. It was found that compounds 7-11 possessed a wide range of anti microbial and anti-HIV1 activity.
\end{abstract}

\section{Introduction}

In spite of the fact that the chemistry quinazolinones have mesmerized the attention of researchers for a long time [1$3]$, the number of competent approaches to the synthesis of their derivatives containing functional groups is inadequate $[4,5]$. The quinazolinone skeleton appears in many alkaloids, most commonly in the form of 4-(3H)-quinazolinone [6]. The quinazolinone moiety is an important pharmacophore showing many types of pharmacological activities. The quinazolinones are considered to be a potent structure for drug developments [7-9]. This has recently inspired the development of a new ring synthesis method. Several successful attempts have been made and recorded in the literature demonstrating promising outcomes [10-12]. The present investigation is a continuation of our earlier [13] study on quinazolinone derivatives.

\section{Result and Discussion}

2.1. Chemistry. In the present investigation, an attempt was made to synthesize quinazolinone derivatives through a multistep process. For this purpose, the required 3(2-aminophenyl)-2-methyl quinazolin-4(3H)-one (1) was prepared according to the literature procedure $[14,15]$, the condensation reaction between benzoxazine and o-phenylene diamine using acetic acid. Formation of the product was confirmed by the formation of intellectual band at $1607 \mathrm{~cm}^{-1}$ (C=N stretching) along with a peak reading at $1699 \mathrm{~cm}^{-1}(\mathrm{C}=\mathrm{O})$ in IR spectra. Benzoxazine [16] was converted to 3(2-aminophenyl)2-methyl quinazolin-4(3H)-one (1) by nucleophilic substitution reaction with o-phenylenediamine along with appearance of new peak near $3398.5 \mathrm{~cm}^{-1}\left(\mathrm{NH}_{2}\right.$ stretching) and $3317.09 \mathrm{~cm}^{-1}$ (N-H stretching) which also helped in assigning structure of (1). When (1) was treated with benzoyl chloride $[17,18]$ in presence of pyridine as a base, nucleophilic reaction took place at the o-phenylenediamine site of molecule, and, as a result, quinazolinone ring was formed, to yield a new heterocyclic compound (2). New band was observed in the IR spectra at $3087.40 \mathrm{~cm}^{-1}$ and a singlet in ${ }^{1} \mathrm{H}$ NMR at $\delta 2.1$ $\left(\mathrm{CH}_{3}\right)$ for methylene protons and a singlet at $8.1-8.19(1 \mathrm{H}, \mathrm{s}$, $\mathrm{CO}-\mathrm{NH} \mathrm{C}-\mathrm{H}$ ) for $\mathrm{NH}$ proton in accordance with the structure of quinazolinone ring. 3-6 compounds were condensed with different aromatic acid chloride derivatives. When compound (2) was treated with phosphorus pentachloride, an intermediate compound was obtained and then further treated with sodium azide which yielded a compound tetrazole moiety (7-11). The bands were observed in IR protons, 
TABLE 1: Antibacterial activity of the quinazolinone compounds at different concentrations.

\begin{tabular}{|c|c|c|c|c|c|c|c|c|c|c|c|c|}
\hline \multirow{3}{*}{ S. no. } & \multirow{3}{*}{ Name of the organisms } & \multicolumn{10}{|c|}{ Zone of inhibition in $\mathrm{mm}$} & \multirow[t]{3}{*}{ STD } \\
\hline & & \multicolumn{2}{|c|}{7} & \multicolumn{2}{|c|}{8} & \multicolumn{2}{|c|}{9} & \multicolumn{2}{|c|}{10} & \multicolumn{2}{|c|}{11} & \\
\hline & & $50 \mu \mathrm{g}$ & $150 \mu \mathrm{g}$ & $50 \mu \mathrm{g}$ & $150 \mu \mathrm{g}$ & $50 \mu \mathrm{g}$ & $150 \mu \mathrm{g}$ & $50 \mu \mathrm{g}$ & $150 \mu \mathrm{g}$ & $50 \mu \mathrm{g}$ & $150 \mu \mathrm{g}$ & \\
\hline (1) & Staphylococcus aureus & 12 & 17 & 11 & 12 & 25 & 20 & 14 & 18 & 16 & 23 & 30 \\
\hline (2) & Escherichia coli & 16 & 19 & 23 & 26 & 20 & 24 & 27 & 29 & 20 & 25 & 38 \\
\hline (3) & Pseudomonas aureus & 20 & 21 & 12 & 14 & 12 & 16 & 17 & 20 & 23 & 28 & 40 \\
\hline$(4)$ & Bacillus subtilis & 16 & 20 & 14 & 13 & 13 & 18 & 15 & 19 & 21 & 23 & 35 \\
\hline
\end{tabular}

Standard (STD)—ciprofloxacin $5 \mu \mathrm{g} /$ disc for bacteria. Solvent control (Sc)—DMSO.

$7.82(17 \mathrm{H}, \mathrm{Ar}-\mathrm{H}$, and $\mathrm{N}=\mathrm{C}=0)$ and $8.57(\mathrm{CH}, \mathrm{Ar}-\mathrm{H})$, respectively, and confirmed the synthesis of final compounds (7-11). The peak values of $1600 \mathrm{~cm}^{-1}\left(\mathrm{C}=\mathrm{N}\right.$ of tetrazole), $1240 \mathrm{~cm}^{-1}$ (tetrazole moiety) compounds at $\delta 2.4\left(\mathrm{CH}_{3}\right)$ for methylene protons were discovered in ${ }^{1} \mathrm{H}$ NMR analysis.

\subsection{Biological Evaluation}

2.2.1. Report on Antimicrobial Activity of the Given Compounds. The antimicrobial activity for the given compounds was carried out by Disc Diffusion Technique (Indian Pharmacopoeia 1996, Volume II A-105). The test microorganisms of Gram-positive Staphylococcus aureus and Gram-negative Escherichia coli, Pseudomonas aereus, and Bacillus subtilis were obtained from National Chemical Laboratory (NCL), Pune, and maintained by periodical subculturing on nutrient agar medium. The effect produced by the sample was compared with that produced by the positive control (reference standard ciprofloxacin $5 \mu \mathrm{g} /$ disc for bacteria).

\subsubsection{Anti-HIV1 Screening}

(1) Cell Culture. The MT-4 cells were grown in RPMI 1640 Dutch Modification (DM) medium, supplemented with 10\% (v/v) heat-inactivated fetal calf serum (FCS), 2 Mm L-glutamine, $0.1 \%$ sodium bicarbonate, and $20 \mu \mathrm{g} / \mathrm{mL}$ gentamycin (equal compete medium). The cells were maintained at $37^{\circ} \mathrm{C}$ in a humidified atmosphere of $5 \% \mathrm{CO}_{2}$ in air. Every 3-4 days and always 2 days before starting the experiment, cells were seeded at $3 \times 10^{5}$ cells $/ \mathrm{mL}$. At regular time intervals, the MT4 cells were analyzed for the presence of mycoplasma and consistently found to be mycoplasma free.

(2) Virus. HIV1 (strain HTLV-IIIB/LAI) was obtained from the culture supernatant of HIV1 infected MT-4 cells lines. The virus titer of the supernatant was determined in MT- 4 cells. The virus stocks were stored at $37^{\circ} \mathrm{C}$ until used.

The present protection achieved by the compound in HIV-infected cells was calculated by the following formula:

$$
\frac{\left(\mathrm{OD}_{\mathrm{T}}\right)_{\mathrm{HIV}}-\left(\mathrm{OD}_{\mathrm{C}}\right)_{\mathrm{HIV}}}{\left(\mathrm{OD}_{\mathrm{C}}\right)_{\mathrm{MOCK}}-\left(\mathrm{OD}_{\mathrm{C}}\right)_{\mathrm{HIV}}} \text { expressed in \%, }
$$

where $\left(\mathrm{OD}_{\mathrm{T}}\right)_{\mathrm{HIV}}$ is the optical density measured with a given concentration of the test compound in HIV-infected cells; $\left(\mathrm{OD}_{\mathrm{C}}\right)_{\mathrm{HIV}}$ is the optical density measured for the control untreated HIV-infected cells; $\left(\mathrm{OD}_{\mathrm{C}}\right)_{\mathrm{MOCK}}$ is the optical
TABle 2: Anti-HIV1 Activity of compounds.

\begin{tabular}{lccc}
\hline Entry & Strain & $\mathrm{IC}_{50}(\mu \mathrm{g} / \mathrm{mL})^{\mathrm{a}}$ & $\mathrm{CC}_{50}(\mu \mathrm{g} / \mathrm{mL})^{\mathrm{b}}$ \\
\hline 7 & $\mathrm{III}_{\mathrm{B}}$ & $>48.9$ & 48.9 \\
8 & $\mathrm{III}_{\mathrm{B}}$ & $>37.5$ & 37.5 \\
9 & $\mathrm{III}_{\mathrm{B}}$ & $>0.456$ & 0.456 \\
10 & $\mathrm{III}_{\mathrm{B}}$ & $>0.482$ & 0.482 \\
11 & $\mathrm{III}_{\mathrm{B}}$ & $>35.1$ & 35.1 \\
$\mathrm{AZT}$ & $\mathrm{III}_{\mathrm{B}}$ & 0.0012 & 65.9 \\
\hline
\end{tabular}

${ }^{a}$ Effective concentration of compounds, achieving $50 \%$ protection of MT- 4 cells against cytopathic effect of HIV.

${ }^{\mathrm{b}}$ Cytotoxic concentration of compounds, required to reduce the viability of mock infected MT- 4 cells by $50 \%$.

density measured for the control untreated HIV-infected cells. The dose achieving 50\% protection according to the above formula was defined as the $50 \%$ effective concentration $\left(\mathrm{EC}_{50}\right)$.

\section{Conclusion}

The present research work demonstrated a genuine and convenient method for synthesizing 3-(2-aminophenyl)-2methyl quinazolin-4(3H) one, N-[(2-(2-methyl-4-oxoquinazolin-3(4H)-1) phenyl)] benzamide, and 2-methyl-3-[(2(5-phenyl-1H-tetrazol-1-yl) phenyl)]-quinazolin-4(3H)-one. The solvent using method for the condensation step was proved to be more proficient and ecofriendly than the traditional procedures. The condensation reaction took place at relatively high temperatures. This method also simplified the handling of the reactions and yielded quinazolin-4(3H)-one derivative. This procedure was simple and low cost. The reactions scheme exhibited moderate activity and valuable contribution to the existing methodologies. The compounds exhibited considerable antibacterial and anti-HIV activities (Tables 1 and 2).

\section{Experimental}

4.1. General Methods. Melting points were uncorrected and recorded on an REMI Series, Lab India Instrument. TLC analysis was done using precoated silica gel plates, and visualization was done using iodine. IR spectra were recorded in $\mathrm{KBr}$ on Shimadzu FT-IR spectrometer. ${ }^{1} \mathrm{H}$ and ${ }^{13} \mathrm{C}-\mathrm{NMR}$ spectra were recorded on a Bruker (AC $400 \mathrm{MHz}$ ) using TMS as an internal standard. Elemental analysis was carried out 
on a Perkin-Elmer series-II CHNS/O Analyzer 2400. All the chemicals were obtained from Aldrich, and all the solvents used were of commercial grade only.

4.2. Synthesis of 3-(2-Aminophenyl)-2-methyl Quinazolin$4(3 \mathrm{H})$ One (1) [12]. An equimolar (0.01 mole) mixture of 2-methyl-3, 1-benzoxazin-4-one and o-phenylenediamine (0.1 mole) was refluxed for $13 \mathrm{~h}$ in the presence of glacial acetic acid $(10 \mathrm{~mL})$. The residue was recrystallized from ethanol and purified by column chromatography to give 1 .

Compound (1): Yield: (74\%); m.p. $218^{\circ} \mathrm{C}$; Anal. Calcd for $\mathrm{C}_{15} \mathrm{H}_{13} \mathrm{~N}_{3} \mathrm{O}$ : C, 71.70; H, 5.21; N, 16.72; O, 6.37; Found: C, 71.74; H, 5.17; N, 16.75; O, 6.32; IR $\left(\mathrm{KBr}, \mathrm{cm}^{-1}\right): 3390.5$ (s, $\mathrm{NH}_{2}$ ), 3310 (s, N-H), 3095 (s, C-H), $1671.3(\mathrm{C}=\mathrm{O}), 1600.48$ (s, C=N); ${ }^{1} \mathrm{H}$ NMR $(400 \mathrm{MHz}$, DMSO-d6, $\delta / \mathrm{ppm}): 8.2(1 \mathrm{H}, \mathrm{s}$, CO-NH C-H), 7.33-7.04 (m, Ar-H), $6.26(\mathrm{C}-\mathrm{H}), 2.14\left(\mathrm{CH}_{3}\right)$; ${ }^{13} \mathrm{C}$ NMR (100 MHz, DMSO-d6, $\left.\delta / \mathrm{ppm}\right): 161$ (Cl-amide), 155.5 (C-2), $148.2(\mathrm{~N}=\mathrm{C}), 145.3(\mathrm{C}-8 \mathrm{a}), 134.5\left(\mathrm{C}-4^{\prime \prime}\right), 127.3$ (C-8), $117.2\left(\mathrm{C}-1^{\prime \prime}\right), 24.3\left(\mathrm{CH}_{3}\right)$; MS $(\mathrm{m} / z$, (relative abundance, \%)): $251.0294(0.10) 237.2923,235$.

4.3. Synthesis of [N-(2-(2-Methyl-4-oxoquinazolin-3(4H)-yl) Phenyl)] Benzamide (2) [12]. The title compound 2 was prepared by using 3-(2-aminophenyl)-2-methyl quinazolin$4(3 \mathrm{H})$ one $(1 ; 0.01$ mole) of an equivalent amount of benzoyl chloride $(0.1 \mathrm{~mole})$ which was refluxed with pyridine $(40 \mathrm{~mL})$ for $11 \mathrm{~h}$. The reaction mixture was cooled, treated with cold ice, and neutralized with conc. $\mathrm{HCl}$. The separated solid was filtered and washed with ice cold water. The residue was recrystallized from ethanol and purified by column chromatography.

Compound (2): Yield: (67\%); m.p. $171^{\circ} \mathrm{C}$; Anal. Calcd for $\mathrm{C}_{22} \mathrm{H}_{17} \mathrm{~N}_{3} \mathrm{O}_{2}$ : C, 74.35; H, 4.82; N, 11.82; O, 9.00; Found: C, 74.30; H, 4.81; N, 11.80; O, 9.02; IR $\left(\mathrm{KBr}, \mathrm{cm}^{-1}\right): 3471.83(\mathrm{~s}$, $\mathrm{N}-\mathrm{H} 1^{\circ}$ amine), 3303 (s, N-H), 3080.40 (s, C-H), 1680.78 (s, $\mathrm{C}=\mathrm{O}), 1609(\mathrm{~s}, \mathrm{C}=\mathrm{N}), 1530$ ( $\mathrm{C}=\mathrm{C}$ aromatic amine), 1270.21 $\left(\mathrm{C}-\mathrm{N}\right.$ str $1^{\circ}$ aromatic amine), $1170(\mathrm{~s}, \mathrm{C}-\mathrm{N}) ;{ }^{1} \mathrm{H}$ NMR (400 MHz, DMSO-d6, $\delta / \mathrm{ppm}$ ): 10.11 (N-H $2^{\circ}$ amide), 8.5-8.3 (Ar-CH), 7.84-7.70 (13H, m, Ar-H), $2.13\left(\mathrm{CH}_{3}\right) ;{ }^{13} \mathrm{C}$ NMR

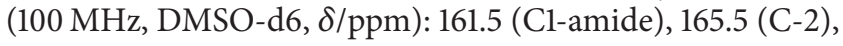
155.5 (C-4), 150 (C-8a), 135.5 (C-1), 130 (C-5), 128.6 (C-6), 127.8 (C-8), $122.6(\mathrm{C}-4 \mathrm{a}), 24.1\left(\mathrm{CH}_{3}\right) ; \mathrm{MS}(\mathrm{m} / z$, (relative abundance, \%)): 355.3978 (0.13), 341.4112, 329.0529.

4.4. Synthesis of 4-Fluoro-[N-(2-(2-methyl-4-oxoquinazolin3(4H)-yl) Phenyl)] Benzamide (3) [12]. The title compound 3 was prepared by using 3-(2-aminophenyl)-2-methyl quinazolin- $4(3 \mathrm{H})$ one $(1 ; 0.01$ mole) of an equivalent amount of 4 fluorobenzoyl chloride ( 0.1 mole) by the general procedure. The separated solid was filtered and washed with ice cold water. The residue was recrystallized from ethanol and purified by column chromatography.

Compound (3): Yield: (63\%); m.p. $162^{\circ} \mathrm{C}$; Anal. Calcd for $\mathrm{C}_{22} \mathrm{H}_{16} \mathrm{FN}_{3} \mathrm{O}_{2}$ : C, 70.77; H, 4.32; N, 11.25; O, 8.57; F, 5.09 Found: C, 70.76; H, 4.36; N, 11.22; O, 8.51; F, 5.07; IR (KBr, $\mathrm{cm}^{-1}$ ): 3474.97 (s, $\mathrm{N}-\mathrm{H} 1^{\circ}$ amine), $3264.19(\mathrm{~s}, \mathrm{~N}-\mathrm{H}), 3070(\mathrm{~s}$, $\mathrm{Ar}-\mathrm{C}-\mathrm{H}), 1680.71(\mathrm{~s}, \mathrm{C}=\mathrm{O}), 1609.24(\mathrm{~s}, \mathrm{C}=\mathrm{N}), 1530.08(\mathrm{C}=\mathrm{C}$ aromatic amine), $1309.26\left(\mathrm{~s}, \mathrm{C}-\mathrm{N}^{\circ}\right.$ aromatic amine), 1167.16 (s, C-N), 1102 (C-F); ${ }^{1} \mathrm{H}$ NMR (400 MHz, DMSO-d6, $\delta / \mathrm{ppm}): 10.29\left(\mathrm{~N}-\mathrm{H} 2^{\circ}\right.$ amide), $8.98(1 \mathrm{H}, \mathrm{s}, \mathrm{H}-\mathrm{C}=\mathrm{N}-\mathrm{N}), 8.18$ $(\mathrm{CH}, \mathrm{F},(\mathrm{C}=\mathrm{O}) \mathrm{N}), 7.87-7.98(13 \mathrm{H}, \mathrm{m}, \mathrm{Ar}-\mathrm{H}),$,7.46 (F subt.), $7.24(\mathrm{CH}, \mathrm{NC}=\mathrm{O}), 2.15\left(\mathrm{CH}_{3}\right) ;{ }^{13} \mathrm{C}$ NMR $(100 \mathrm{MHz}$, DMSOd6, $\delta / \mathrm{ppm}): 161.5$ (Cl-amide), $165.6(\mathrm{H}-\mathrm{C}=\mathrm{N}), 167.4(=\mathrm{C}(\mathrm{F})$, C-4" $\left.{ }^{\prime \prime}\right), 155.2(\mathrm{C}-2), 147.8(\mathrm{C}-8 \mathrm{a}), 134.3(\mathrm{C}-7), 131.4\left(\mathrm{C}-1^{\prime \prime}\right), 129.8$ $\left(\mathrm{C}-2^{\prime}\right), 128.4(\mathrm{C}-6), 127.6$ (C-8), 122.6 (C-4a), $118.5\left(\mathrm{C}-3^{\prime \prime}\right), 24.1$ $\left(\mathrm{CH}_{3}\right)$; MS $(\mathrm{m} / \mathrm{z}$, (relative abundance, \%)): $375.2454(0.13)$, 355.1354, 299.1021 .

4.5. Synthesis of 4-Cyano-[N-(2-(2-methyl-4-oxoquinazolin3(4H)-yl) Phenyl)] Benzamide (4) [13]. The title compound 4 was prepared by using 3-(2-aminophenyl)-2-methyl quinazolin-4(3H) one (1; 0.01 mole) of an equivalent amount of 4cyanobenzoyl chloride $(0.1$ mole) by the general procedure. The separated solid was filtered and washed with ice cold water. The residue was recrystallized from ethanol and purified by column chromatography.

Compound (4): Yield: (66\%); m.p. $165^{\circ} \mathrm{C}$; Anal. Calcd for $\mathrm{C}_{23} \mathrm{H}_{16} \mathrm{~N}_{4} \mathrm{O}_{2}$ : C, 72.62; H, 4.24; N, 14.73; O, 8.41; Found: C, $72.60 ; \mathrm{H}, 4.26 ; \mathrm{N}, 14.77$; O, 8.40; IR $\left(\mathrm{KBr}, \mathrm{cm}^{-1}\right): 3505(\mathrm{~s}, \mathrm{~N}-$

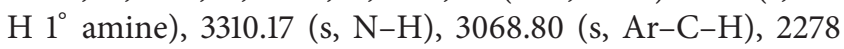
$(\mathrm{C} \equiv \mathrm{N}), 1679.16(\mathrm{~s}, \mathrm{C}=\mathrm{O}), 1600.27(\mathrm{~s}, \mathrm{C}=\mathrm{N}), 1540.46(\mathrm{C}=\mathrm{C}$ aromatic amine), 1298.15 (s, C-N), 1177 (s, C-N); ${ }^{1} \mathrm{H}$ NMR (400 MHz, DMSO-d6, $\delta / \mathrm{ppm}$ ): 10.29 (N-H $2^{\circ}$ amide), 8.99 $(1 \mathrm{H}, \mathrm{s}, \mathrm{H}-\mathrm{C}=\mathrm{N}-\mathrm{N}), 8.13-8.45(\mathrm{CH}, \mathrm{CN},(\mathrm{C}=\mathrm{O}) \mathrm{N}), 7.80-7.99$ $(13 \mathrm{H}, \mathrm{m}, \mathrm{Ar}-\mathrm{H}),, 7.27(\mathrm{CH}, \mathrm{NC}=\mathrm{O}), 2.15\left(\mathrm{CH}_{3}\right),{ }^{13} \mathrm{C}$ NMR (100 MHz, DMSO-d6, $\delta / \mathrm{ppm}): 161.5$ (C1-amide), 165.6 (H$\mathrm{C}=\mathrm{N}), 155.2(\mathrm{C}-2), 139.8(\mathrm{C}-8 \mathrm{a}), 132.9(\mathrm{C}-4), 130.9\left(\mathrm{C}-1^{\prime \prime}\right)$, $129.9\left(\mathrm{C}-2^{\prime}\right), 128.8((\mathrm{C}=\mathrm{O})-\mathrm{N}, \mathrm{CN}), 127.2(\mathrm{C}-8), 121.6(\mathrm{C}-4 \mathrm{a})$, 118.5 (nitrile), $24.1\left(\mathrm{CH}_{3}\right) ; \mathrm{MS}(\mathrm{m} / z$, (relative abundance, \%)): 381.2336 (0.18), 367.1277, 355.1343, 305.1722 (0.06).

4.6. Synthesis of 4-Chloro-[N-(2-(2-methyl-4-oxoquinazolin3(4H)-yl) Phenyl)] Benzamide (5) [13]. The title compound 5 was prepared by using 3-(2-aminophenyl)-2-methyl quinazolin- $4(3 \mathrm{H})$ one $(1 ; 0.01$ mole) of an equivalent amount of 4 chlorobenzoyl chloride $(0.1$ mole) by the general procedure. The separated solid was filtered and washed with ice cold water. The residue was recrystallized from ethanol and purified by column chromatography.

Compound (5): Yield: (61\%); m.p. $174^{\circ} \mathrm{C}$; Anal. Calcd for $\mathrm{C}_{22} \mathrm{H}_{16} \mathrm{ClN}_{3} \mathrm{O}_{2}$ : C, 67.78; H, 4.14; N, 10.78; O, 8.21; Cl, 9.09; Found: C, 67.74; H, 4.18; N, 10.73; O, 8.20; Cl, 9.05; IR (KBr, $\left.\mathrm{cm}^{-1}\right): 3471.92\left(\mathrm{~s}, \mathrm{~N}-\mathrm{H} 1^{\circ}\right.$ amine), $3296.43(\mathrm{~s}, \mathrm{~N}-\mathrm{H}), 3068.98$ (s, Ar-C-H), 1677.70 (s, C=O), 1596.39 (s, C=N), 1535.61 $\left(\mathrm{C}=\mathrm{C}\right.$ aromatic amine), $1330.05\left(\mathrm{C}-\mathrm{N}\right.$ str $1^{\circ}$ aromatic amine), 1170 (s, C-N), 752.46 (C-Cl); ${ }^{1} \mathrm{H}$ NMR (400 MHz, DMSO-d6, $\delta / \mathrm{ppm}): 10.29\left(\mathrm{~N}-\mathrm{H} 2^{\circ}\right.$ amide), 8.27-8.1 ( $\left.\mathrm{Ar}-\mathrm{CH}\right), 7.84-7.99$ $(13 \mathrm{H}, \mathrm{m}, \mathrm{Ar}-\mathrm{H}, \mathrm{Cl}), 7.78(\mathrm{Cl}$ subt.), $7.40(\mathrm{CH}, \mathrm{NC}=\mathrm{O}), 2.14$ $\left(\mathrm{CH}_{3}\right) ;{ }^{13} \mathrm{C}$ NMR $(100 \mathrm{MHz}$, DMSO-d6, $\delta / \mathrm{ppm}): 161.4$ (C1amide), $165.9(\mathrm{H}-\mathrm{C}=\mathrm{N}), 155$ (C-2), 147.6 (C-8a), 140, 138.9, 134.8 (C-7), 132, 130.6 (C-2", $\mathrm{Cl}), 129.7$ (C-3'), 127.9 (C-6), $126.8(\mathrm{C}-8), 120.9(\mathrm{C}-4 \mathrm{a}), 24.1\left(\mathrm{CH}_{3}\right)$; $\mathrm{MS}(\mathrm{m} / \mathrm{z}$, (relative abundance, \%)): 391.4036 (0.10), 377.0915, 355.2139, 329.1816.

4.7. Synthesis of [N-(2-(2-Methyl-4-oxoquinazolin-3(4H)-yl) Phenyl)]-4-nitrobenzamide (6) [13]. The title compound 6 
was prepared by using 3-(2-aminophenyl)-2-methyl quinazolin- $4(3 \mathrm{H})$ one $(1 ; 0.01$ mole $)$ of an equivalent amount of 4 -nitrobenzoyl chloride $(0.1$ mole) by the general procedure. The separated solid was filtered and washed with ice cold water. The residue was recrystallized from ethanol and purified by column chromatography.

Compound (6): Yield: (74\%); m.p. $245^{\circ} \mathrm{C}$; Anal. Calcd for $\mathrm{C}_{22} \mathrm{H}_{16} \mathrm{~N}_{4} \mathrm{O}_{4}$ : C, 66.00; H, 4.03; N, 13.99; O, 15.98; Found: C, 66.09; H, 4.01; N, 13.95; O, 15.94; IR $\left(\mathrm{KBr}, \mathrm{cm}^{-1}\right)$ : 3501 (s, N$\mathrm{H} 1^{\circ}$ amine), 3319.26 (s, N-H), 3088.86 (s, Ar-C-H), 1690 (s, $\mathrm{C}=\mathrm{O}), 1600.14(\mathrm{~s}, \mathrm{C}=\mathrm{N}), 1499(\mathrm{C}=\mathrm{C}$ aromatic amine $), 1300.33$ $\left(\mathrm{C}-\mathrm{N}\right.$ str $1^{\circ}$ aromatic amine), $1345\left(\mathrm{Ar}-\mathrm{NO}_{2}\right), 1180$ (s, C$\mathrm{N}) ;{ }^{1} \mathrm{H}$ NMR (400 MHz, DMSO-d6, $\left.\delta / \mathrm{ppm}\right): 10.28\left(\mathrm{~N}-\mathrm{H} 2^{\circ}\right.$ amide), $8.20-8.80(\mathrm{~s}, \mathrm{H}-\mathrm{C}=\mathrm{N})(\mathrm{CH},(\mathrm{N}=\mathrm{O})=\mathrm{O},(\mathrm{C}=\mathrm{O})-\mathrm{N})$, $7.99(\mathrm{H}-8, \mathrm{~m}, \mathrm{Ar}-\mathrm{H}, \mathrm{Cl}), 7.65\left(\mathrm{H}-4^{\prime} / \mathrm{H}-4^{\prime \prime}\right), 7.30(\mathrm{CH}, \mathrm{NC}=\mathrm{O}$, $\mathrm{H}-7), 2.12\left(\mathrm{CH}_{3}\right) ;{ }^{13} \mathrm{C}$ NMR (100 MHz, DMSO-d6, $\left.\delta / \mathrm{ppm}\right)$ : 161.5 (C1-amide), 167.5 (C-2), 157, $151.5((\mathrm{~N}=\mathrm{O})=\mathrm{O},(\mathrm{C}=\mathrm{O})-$ $\mathrm{N}), 135\left(=\mathrm{C}-\left(\mathrm{NO}_{3}\right), \mathrm{C}-3^{\prime \prime}\right), 134\left(\mathrm{C}-1^{\prime \prime}\right), 131.2(\mathrm{C}-7), 129.9(\mathrm{C}-$ $\left.2^{\prime}\right), 128.5$ (C-6), 127.5 (C-8), 122.6 (C-4a), $116\left(\mathrm{C}-3^{\prime \prime}\right), 24.2$ $\left(\mathrm{CH}_{3}\right)$; MS ( $\mathrm{m} / \mathrm{z}$, (relative abundance, \%)): $401.2235(0.15)$, 399, 387.3 .

4.8. Synthesis of 2-Methyl-3-(2-(5-phenyl-1H-tetrazol-1-yl) Phenyl) Quinazolin-4(3H)-one (7) [13]. The title compound 7 was prepared by using [N-(2-(2-methyl-4-oxoquinazolin$3(4 \mathrm{H})$-yl) phenyl)] benzamide $(2 ; 0.01$ mole) which was taken in a beaker, added to a known amount of PCl5 (0.01 mole), and heated at $100^{\circ} \mathrm{C}$ until the evaluation of $\mathrm{HCl}$ fumes ceased. The reaction mixtures contained some unreacted $\mathrm{POCl}_{3}$ which was removed by distillation under reduced pressure. The resulting mixture was treated with ice cold solution of known weight of $\mathrm{NaN}_{3}$ (0.02 moles); a known volume $(40 \mathrm{~mL})$ of acetone and known volume of sodium acetate were added. The reaction mixture was stirred for $30 \mathrm{~h}$. The acetone was removed by distillation under reduced pressure. The resulting mixture was extracted with $\mathrm{CHCl}_{3}$; then the organic layer was separated and evaporated; we got product. The product filtered and washed with ice cold water. The residue was recrystallized from benzene-pet-ether mixture and purified by column chromatography to give 7 .

Compound (7): Yield: (50\%); m.p. $254^{\circ}$ C; Anal. Calcd for $\mathrm{C}_{22} \mathrm{H}_{16} \mathrm{~N}_{6} \mathrm{O}$ : C, 69.46; H, 4.24; N, 22.09; O, 4.21; Found: C, 69.41; H, 4.29; N, 22.07; O, 4.20; IR $\left(\mathrm{KBr}, \mathrm{cm}^{-1}\right)$ : 3300 (s, N$\mathrm{H}), 3060$ (s, Ar-C-H), $1658(\mathrm{~s}, \mathrm{C}=\mathrm{N}), 1600(\mathrm{C}=\mathrm{N}$, tetrazole), 1240 (tetrazole, $\mathrm{N}-\mathrm{N}=\mathrm{N}) ;{ }^{1} \mathrm{H}$ NMR $(400 \mathrm{MHz}, \mathrm{DMSO}-\mathrm{d} 6$, $\delta / \mathrm{ppm}): 8.57,8.6,8.9,9.5(\mathrm{CH}, \mathrm{Ar}-\mathrm{H}), 7.82(17 \mathrm{H}, \mathrm{Ar}-\mathrm{H}$, $\mathrm{N}=\mathrm{C}=0), 7.70(13 \mathrm{H}, \mathrm{Ar}-\mathrm{H},(\mathrm{C}=\mathrm{O}) \mathrm{N}), 2.4\left(\mathrm{CH}_{3}\right) ;{ }^{13} \mathrm{C} \mathrm{NMR}$

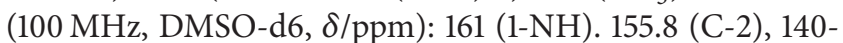
147.3 (C-8a), 135 (C-4' ), 133, $131\left(\mathrm{C}-3^{\prime}\right), 129$ (C-6), 127.8 (C-2'), $125.6(\mathrm{C}-8), 123\left(\mathrm{C}-1^{\prime \prime}\right), 121.7(\mathrm{C}-4 \mathrm{a}), 24.9\left(\mathrm{CH}_{3}\right)$; $\mathrm{MS}(\mathrm{m} / z$, (relative abundance, \%)): 381.2453 (0.31), 367.0372, 353.01347, 305.1032.

4.9. Synthesis of 3-(2-(5-(4-Fluorophenyl)-1H-tetrazol-1-yl) Phenyl)-2-methyl Quinazolin-4(3H)-one (8) [13]. The title compound 8 was prepared by using 4-fluoro-[N-(2-(2-methyl-4oxoquinazolin-3(4H)-yl) phenyl)] benzamide (2; 0.01 mole) by the general procedure; the residue was recrystallized from benzene-pet-ether mixture and purified by column chromatography.

Compound (8): Yield: (51\%); m.p. $262^{\circ} \mathrm{C}$; Anal. Calcd for $\mathrm{C}_{22} \mathrm{H}_{15} \mathrm{FN}_{6} \mathrm{O}$ : C, 66.33; H, 3.80; N, 21.09; O, 4.02; F, 4.77; Found: C, 66.36; H, 3.83; N, 21.04; O, 4.01; IR (KBr, $\mathrm{cm}^{-1}$ ): $3315.66(\mathrm{~N}-\mathrm{H}$ stretching), 3070.79 (aromatic $\mathrm{C}-\mathrm{H}$ stretching), 1660.62 ( $\mathrm{C}=\mathrm{N}$ stretching), $1605(\mathrm{C}=\mathrm{N}$, tetrazole), 1107 (C-F), 1259 (tetrazole, N-N=N); ${ }^{1} \mathrm{H}$ NMR $(400 \mathrm{MHz}$, DMSO-d6, $\delta / \mathrm{ppm}$ ): 8.9 (H-5), 7.88 (F, C-Ar), 7.82 (17H, Ar$\mathrm{H}, \mathrm{N}=\mathrm{C}=0), 7.70(13 \mathrm{H}, \mathrm{Ar}-\mathrm{H},(\mathrm{C}=\mathrm{O}) \mathrm{N}), 7.37(\mathrm{~F}, \mathrm{C}-\mathrm{Ar}), 2.3$ $\left(\mathrm{CH}_{3}\right) ;{ }^{13} \mathrm{C}$ NMR $(100 \mathrm{MHz}, \mathrm{DMSO}-\mathrm{d} 6, \delta / \mathrm{ppm}): 164.4$ (1-F, 1-Ar), 155.7 (C-2), 146.3 (C-8a), 135.9 (C1, C-Ar), 130.6 (C-6), 129.2 (C1, C-Ar), 128 (C-2' $), 126.8,125,124.7$ (F, 1-Ar), 121.6 (C-4a), $117.2\left(\mathrm{CH}_{1}-\mathrm{F}\right), 25\left(\mathrm{CH}_{3}\right) ; \mathrm{MS}(\mathrm{m} / z$, (relative abundance, \%)): 399.3438 (0.24), 385.010, 381.1219, 371.1526, 323.0922 .

4.10. Synthesis of 4-(1-(2-(2-Methyl-4-oxoquinazolin-3(4H)-yl) Phenyl)-1H-tetrazol-5-yl) Benzonitrile (9) [13]. The title compound 9 was prepared by using 4-cyano-[N-(2-(2-methyl-4oxoquinazolin-3(4H)-yl) phenyl)] benzamide (2; 0.01 mole) by the general procedure; the residue was recrystallized from benzene-pet-ether mixture and purified by column chromatography.

Compound (9): Yield: (53\%); m.p. $267^{\circ} \mathrm{C}$; Anal. Calcd for $\mathrm{C}_{23} \mathrm{H}_{15} \mathrm{~N}_{7} \mathrm{O}$ : C, 68.14; H, 3.73; N, 24.18; O, 3.95; Found: C, 68.11; H, 3.76; N, 24.15; O, 3.98; IR $\left(\mathrm{KBr}, \mathrm{cm}^{-1}\right): 3000$ (s, N$\mathrm{H}), 3083$ (s, Ar-C-H), 1698.39 (s, C=O), 1610.62 (tetrazole), 1500.08 ( $\mathrm{C}=\mathrm{C}$ aromatic amine), 1307.59 (s, $\mathrm{C}-\mathrm{N} 1^{\circ}$ aromatic amine), 1280.84 (tetrazole, $\mathrm{N}-\mathrm{N}=\mathrm{N}), 1188.97(\mathrm{~s}, \mathrm{C}-\mathrm{N}) ;{ }^{1} \mathrm{H}$ NMR (400 MHz, DMSO-d6, $\delta / \mathrm{ppm}): 8.2-8.6(\mathrm{CH},(\mathrm{C}=\mathrm{O}) \mathrm{N})$, 7.99 (CN, Ar-H), $2.3\left(\mathrm{CH}_{3}\right) ;{ }^{13} \mathrm{C}$ NMR (100 MHz, DMSO-d6, $\delta / \mathrm{ppm}): 161(\mathrm{H}-\mathrm{C}=\mathrm{N}), 155.7(\mathrm{C}-2), 139.8(\mathrm{C}-8 \mathrm{a}), 135.7(\mathrm{CN}$, Ar-C), 130 (C-6), 129, 128.3 (C1, C-Ar), 126.6 (C-8), 121.6 (C4a), 119.2 (nitrile), $114(\mathrm{CN}), 25.5\left(\mathrm{CH}_{3}\right)$; MS $(\mathrm{m} / z$, (relative abundance, \%)): 405.3214 (0.32), 391.2133, 381.0310, 331.1377.

4.11. Synthesis of 3-(2-(5-(4-Chlorophenyl)-1H-tetrazol-1-yl) Phenyl)-2-methyl Quinazolin-4(3H)-one (10) [13]. The title compound 10 was prepared by using 4-chloro-[N-(2-(2-methyl-4oxoquinazolin-3(4H)-yl) phenyl)] benzamide (2; 0.01 mole) by the general procedure; the residue was recrystallized from benzene-pet-ether mixture and purified by column chromatography.

Compound (10): Yield: (50\%); m.p. $278^{\circ} \mathrm{C}$; Anal. Calcd for $\mathrm{C}_{22} \mathrm{H}_{15} \mathrm{ClN}_{6} \mathrm{O}$ : C, 63.69; H, 3.64; N, 20.26; O, 3.86; Cl, 8.55 Found: C, 63.66; H, 3.67; N, 20.28; O, 3.88; Cl, 8.54; IR ( $\mathrm{KBr}$, $\mathrm{cm}^{-1}$ ): 3290 (N-H stretching), 3066.24 (Ar-H stretching), $1655(\mathrm{C}=\mathrm{N}$ stretching), $1600(\mathrm{C}=\mathrm{N}$, tetrazole), 1240 (tetrazole, $\mathrm{N}-\mathrm{N}=\mathrm{N}$ ), 789 (C-Cl); ${ }^{1} \mathrm{H}$ NMR (400 MHz, DMSO-d6, $\delta / \mathrm{ppm}):$ 8.20, 8.46 (1-Cl, Ar-H), $7.82(17 \mathrm{H}, \mathrm{Ar}-\mathrm{H}, \mathrm{N}=\mathrm{C}=0)$, $7.70(13 \mathrm{H}, \mathrm{Ar}-\mathrm{H},(\mathrm{C}=\mathrm{O}) \mathrm{N}), 7.50(\mathrm{Cl}, \mathrm{C}-\mathrm{Ar}), 2.3\left(\mathrm{CH}_{3}\right) ;{ }^{13} \mathrm{C}$

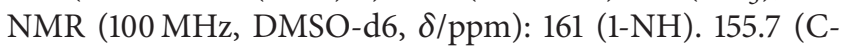
2), 148.3 (C-8a), 135.2 (Cl, C-Ar), 129-129.9 (C-6), 128.6-129 (Cl, C-Ar), 127 (C-2' ), $126.4(\mathrm{C}-8), 121.6 \mathrm{C}-4 \mathrm{a}), 25\left(\mathrm{CH}_{3}\right)$; $\mathrm{MS}$ $(\mathrm{m} / z$, (relative abundance, \%)): $417.2136(0.80), 401.1726$, 387.1813, 381.1511, 341.0947. 
4.12. Synthesis of 2-Methyl-3-(2-(5-(4-nitrophenyl)-1H-tetrazol-1-yl) Phenyl) Quinazolin-4(3H)-one (11) [13]. The title compound 11 was prepared by using [N-(2-(2-methyl-4oxoquinazolin-3(4H)-yl) phenyl)]-4-nitrobenzamide (2; 0.01 mole) by the general procedure; the residue was recrystallized from benzene-pet-ether mixture and purified by column chromatography.

Compound (11): Yield: (52\%); m.p. $280^{\circ} \mathrm{C}$; Anal. Calcd for $\mathrm{C}_{22} \mathrm{H}_{15} \mathrm{~N}_{7} \mathrm{O}_{3}$ : C, 62.11; H, 3.55; N, 23.05; O, 11.28; Found: C, 62.16; H, 3.59; N, 23.09; O, 11.24; IR $\left(\mathrm{KBr}, \mathrm{cm}^{-1}\right): 3292$ (s, N$\mathrm{H}), 3043.50(\mathrm{~s}, \mathrm{C}-\mathrm{H}), 1700.52(\mathrm{~s}, \mathrm{C}=\mathrm{O}), 1608.28(\mathrm{C}=\mathrm{N}$, tetrazole), 1507.07 ( $\mathrm{C}=\mathrm{C}$ aromatic amine), $1320\left(\mathrm{~s}, \mathrm{C}-\mathrm{N}^{\circ}\right.$ aromatic amine), $1360\left(\mathrm{Ar}-\mathrm{NO}_{2}\right), 1162(\mathrm{~s}, \mathrm{C}-\mathrm{N}) ;{ }^{1} \mathrm{H}$ NMR $(400 \mathrm{MHz}$, DMSO-d6, $\delta / \mathrm{ppm}): 8.2-8.76\left(\mathrm{NO}_{2}\right), 7.90(\mathrm{H}-8, \mathrm{NC}=\mathrm{O}), 7.85$ $(\mathrm{CH},(\mathrm{C}=\mathrm{O}) \mathrm{N}), 2.77\left(\mathrm{CH}_{3}\right) \cdot{ }^{13} \mathrm{C}$ NMR $(100 \mathrm{MHz}$, DMSO-d6, $\delta / \mathrm{ppm}): 161$ (C1-amide), 155.8 (C-2), 150.2 (C-8a), 140.6 (C8a), $138.4\left(\mathrm{NO}_{2}\right), 129.7\left(\mathrm{C}-2^{\prime}\right), 127.8\left(\mathrm{CH}, \mathrm{NO}_{2}\right), 126.8(\mathrm{C}-8)$, $125.7\left(\mathrm{NO}_{2}, \mathrm{CH}\right), 125.9(\mathrm{~N}-\mathrm{C}=\mathrm{O}), 122.6(\mathrm{C}-4 \mathrm{a}), 25\left(\mathrm{CH}_{3}\right) ; \mathrm{MS}$ $(\mathrm{m} / \mathrm{z}$, (relative abundance, \%)): $425.1445(0.16), 411.1235$, 399.1528, 381.1412, 351.1177.

\section{Acknowledgments}

The authors are thankful to the Principal and Management Committee members of Jamal Mohamed College, Srimad Andavan Arts and Science College, and UGC, New Delhi, for providing necessity facilities for this work.

\section{References}

[1] M. A. Saleh, M. F. Abdel-Megeed, M. A. Abdo, and A. M. Shokr, "Synthesis of novel 3H-quinazolin-4-ones containing pyrazolinone, pyrazole and pyrimidinone moieties," Molecules, vol. 8, no. 4, pp. 363-373, 2003.

[2] M. A. Sayyed, S. S. Mokle, and Y. B. Vibhute, "Synthesis of 6-iodo/bromo- 3-amino-2-methylquinazolin-4 (3H)-ones by direct halogenation and their Schiff base derivatives," Arkivoc, vol. 2006, no. 11, pp. 221-226, 2006.

[3] G. Ouyang, P. Zhang, G. Xu et al., "Synthesis and antifungal bioactivities of 3-alkylquinazolin-4-one derivatives," Molecules, vol. 11, no. 6, pp. 383-392, 2006.

[4] V. Alagarsamy, V. R. Salomon, G. Vanikavitha et al., "Synthesis, analgesic, anti-inflammatory and antibacterial activities of some novel 2-phenyl-3-substituted quinazolin-4(3H) ones," Biological and Pharmaceutical Bulletin, vol. 25, no. 11, pp. 14321435, 2002.

[5] G. Liu, S. Yang, B. Song et al., "Microwave assisted synthesis of $\mathrm{N}$-arylheterocyclic substituted-4-aminoquinazoline derivatives," Molecules, vol. 11, no. 4, pp. 272-278, 2006.

[6] U. Ameta, S. Ojha, D. Bhambi, and G. L. Talesara, "Synthetic studies on some 3-[(5-arylidene-4-oxo-1,3-thiazolidin-2-yliden) amino]-2-phenylquinazolin-4(3H)-ones and their ethoxyphthalimide derivatives," Arkivoc, vol. 2006, no. 13, pp. 83-89, 2006.

[7] A. K. Nanda, S. Ganguli, and R. Chakraborty, "Antibacterial activity of some 3-(arylideneamino)-2-phenylquinazoline4(3H)-ones: synthesis and preliminary QSAR studies," Molecules, vol. 12, no. 10, pp. 2413-2426, 2007.

[8] P. M. Chandrika, T. Yakaiah, B. Narsaiah et al., "Synthesis leading to novel 2,4,6-trisubstituted quinazoline derivatives, their antibacterial and cytotoxic activity against thp-1, hl-60 and a375 cell lines," Indian Journal of Chemistry B, vol. 48, no. 6, pp. 840 $847,2009$.

[9] O. A. M. . Fathalla, E. M. M. Kassem, and N. M. Ibrahem, "Synthesis of some new quinazolin-4-one derivatives and evaluation of their antimicrobial and anti-inflammatory effects," Acta Poloniae Pharmaceutican Drug Research, vol. 65, no. 1, pp. 11-20, 2008.

[10] H. P. Shah, B. R. Shah, J. J. Bhatt, N. C. Desai, P. B. Trivedi, and N. K. Undavia, "Synthesis of 2,5-disubstituted 1,3,4-oxadiazoles as potential antimicrobial, anticancer and anti-HIV agents," Indian Journal of Chemistry B, vol. 37, no. 2, pp. 180-182, 1998.

[11] V. Alagarswamy, U. S. Pathak, S. N. Pandaya, D. Sriram, and E. De Clercq, "Anti HIV and antibacterial activities of some disubstitutedquinazolones and their bio-isostere disubstitutedthienopyrimidones," Indian Journal of Pharmaceutical Sciences, vol. 62, p. 433, 2000.

[12] M. K. Srivastava, B. Misra, and N. Nizamuddin, "Pharmacological studies of some 2-methyl-3-(arylthio-carbamido) quinazol4-ones and 2-methyl-3-(aryliden-carboxamido) quinazol-4ones," Indian Journal of 8 Chemistry B, vol. 40, p. 342, 2001.

[13] K. Vijayakumar and A. Jafar Ahamed, "Synthesis and biological activities of some novel substituted quinazoline derivatives," Der Pharma Chemica, vol. 2, no. 5, p. 453, 2010.

[14] L. A. Errede, J. J. McBrady, and H. T. Oien, "Acylanthranils. 2. The problem of selectivity in the reaction of acetylanthranil with anilines," Journal of Organic Chemistry, vol. 41, no. 10, pp. 17651768, 1976.

[15] G. M. El-Naggar, H. A. H. El-Sherief, K. Aref, and H. AbdelHamid, "Synthesis of some new 6-bromo-2-methyl-3-(p-substituted aminophenyl)-4(3H)-quinazolinones," Pharmazie, vol. 38, p. 821, 1983.

[16] V. Jatav, P. Mishra, S. Kashaw, and J. P. Stables, "Synthesis and CNS depressant activity of some novel 3-[5-substituted 1,3,4thiadiazole-2-yl]-2-styryl quinazoline-4(3H)-ones," European Journal of Medicinal Chemistry, vol. 43, no. 1, pp. 135-141, 2008.

[17] A. Al-Omar, S. Adel El-Azab, H. A. El-Obeid, and S. G. Abdel Hamid, "Synthesis of some new 4-(3H)-quinazolinee analouges as potential antioxidant agentes," Journal of Saudi Chemical Society, vol. 10, p. 113, 2006.

[18] O. A. E. M. Fathalla, E. M. M. Kassem, N. M. Ibrahem, and M. M. Kamel, "Synthesis of some new quinazolin-4-one derivatives and evaluation of their antimicrobial and antiinflammatory effects," Acta Poloniae Pharmaceutica, vol. 65, no. 1, pp. 11-20, 2008. 

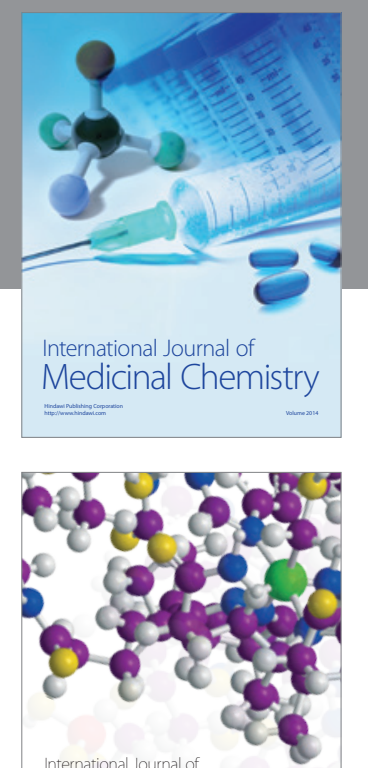

\section{Carbohydrate} Chemistry

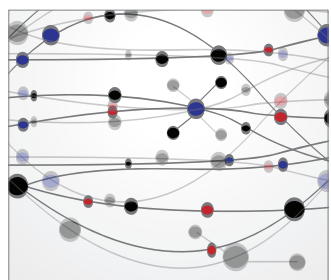

The Scientific World Journal
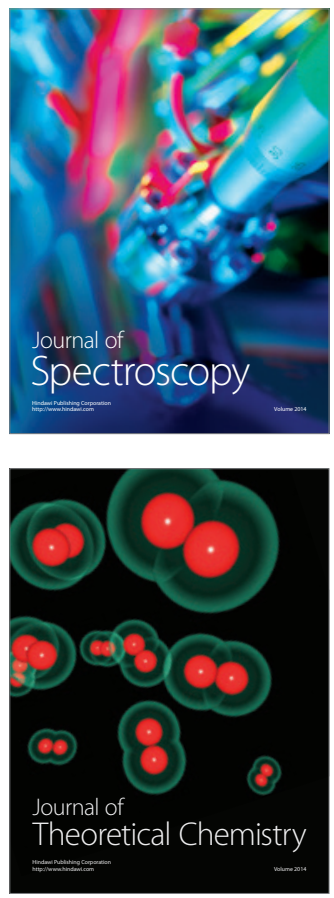
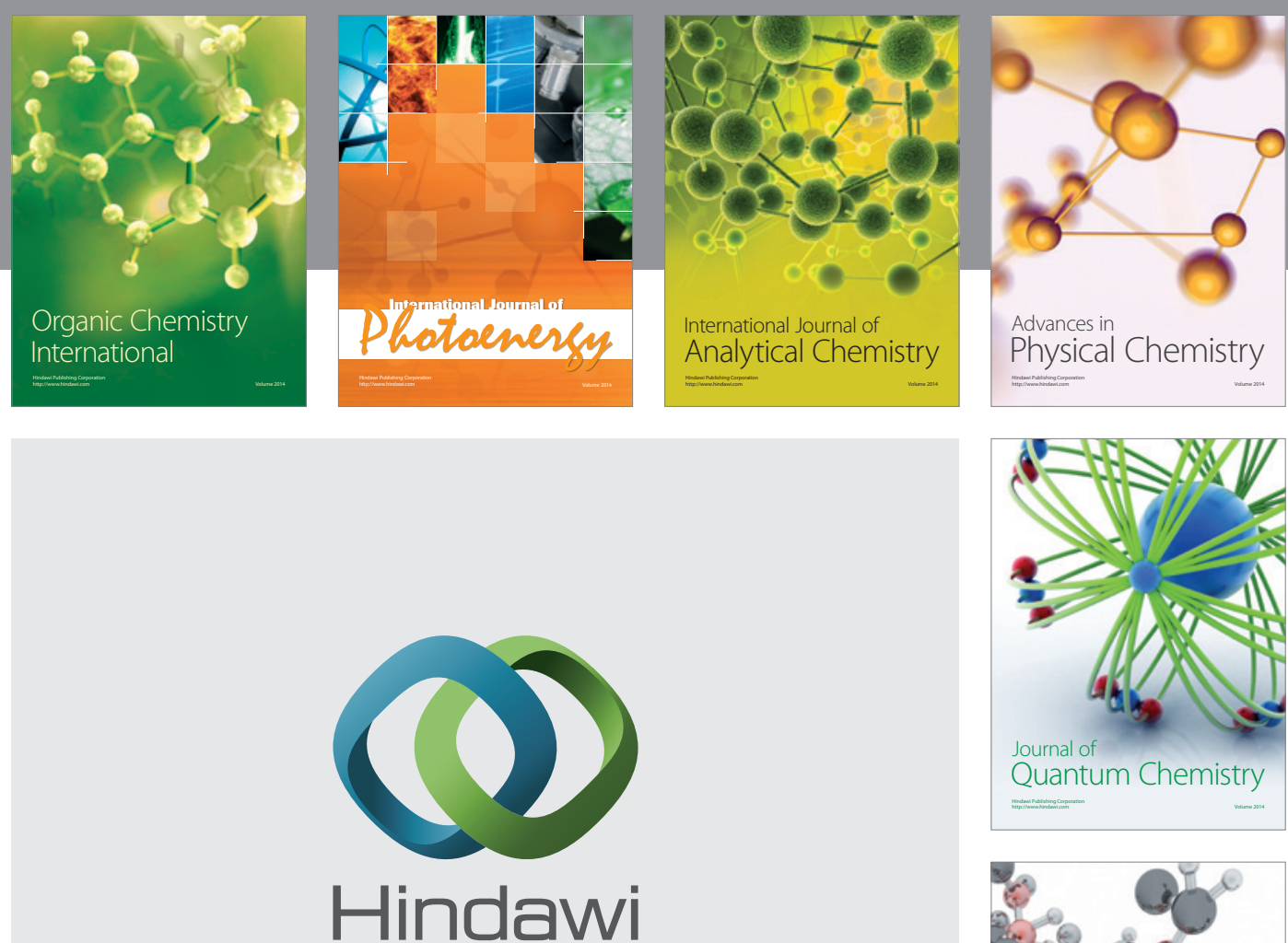

Submit your manuscripts at

http://www.hindawi.com

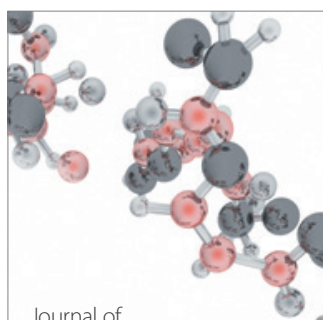

Analytical Methods

in Chemistry

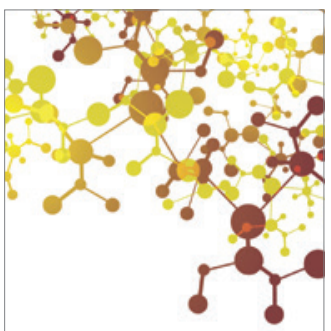

Journal of

Applied Chemistry

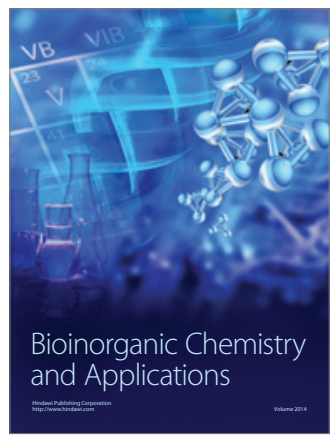

Inorganic Chemistry
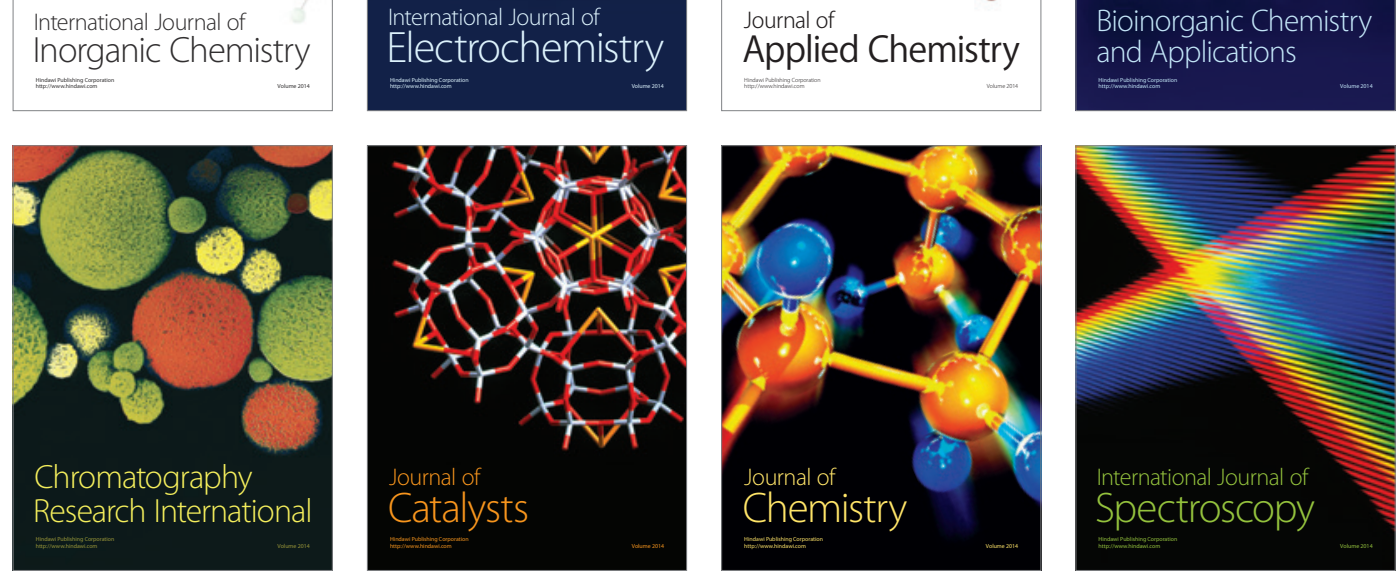